\title{
CONFORT CLIMÁTICO Y TURISMO. ESTUDIO APLICADO A DIFERENTES NIVELES DE RESOLUCIÓN TEMPORAL EN PUERTO IGUAZÚ, ARGENTINA
}

\author{
Ariadna Belén Tanana \\ Departamento de Geografía y Turismo - Universidad Nacional del Sur (DGyT - UNS); Consejo Nacional de Investigaciones \\ Científicas y Técnicas (CONICET) \\ ariadna.tanana@uns.edu.ar \\ ORCID ID: https://orcid.org/0000-0002-9995-647X \\ María Belén Ramos \\ Departamento de Geografía y Turismo - Universidad Nacional del Sur (DGyT - UNS) \\ mberamos@uns.edu.ar \\ ORCID ID: https://orcid.org/0000-0002-6420-354X \\ Verónica Gil \\ Departamento de Geografía y Turismo - Universidad Nacional del Sur (DGyT - UNS); Consejo Nacional de Investigaciones \\ Científicas y Técnicas (CONICET) \\ verogil@uns.edu.ar \\ ORCID ID: https://orcid.org/0000-0002-2824-204X \\ Alicia María Campo \\ Departamento de Geografía y Turismo - Universidad Nacional del Sur (DGyT - UNS) Consejo Nacional de Investigaciones \\ Científicas y Técnicas (CONICET) \\ amcampo@uns.edu.ar \\ ORCID ID: https://orcid.org/0000-0003-1150-4039
}

Recibido: 11/06/2019; Aceptado: 24/03/2021; Publicado: 22/06/2021

Cómo citar este artículo/citation: Tanana, Ariadna Belén; Ramos, María Belén; Gil, Verónica y Campo, Alicia María (2021). Confort
climático y turismo. Estudio aplicado a diferentes niveles de resolución temporal en Puerto Iguazú, Argentina. Estudios Geográficos, 82 (290), e064. https://doi.org/10.3989/estgeogr.202076.076

RESUMEN: El clima es un factor que condiciona las prácticas turístico-recreativas en espacios al aire libre. El bienestar y confort térmico constituyen exigencias del visitante susceptibles de evaluarse mediante la faceta térmica del clima. Este trabajo se aplica a Puerto Iguazú, un destino de clima subtropical húmedo. El mismo está estructurado con base en dos objetivos, por un lado, comparar tres índices bioclimáticos: Complejo Termo-anemométrico (P), Complejo Termo-higrométrico (THI) y Humidex (H), versus un índice climático-turístico: Tourism Climate Index $(\mathrm{TCl})$ a fin de establecer cuál ofrece un mejor ajuste a la realidad estudiada. Por otro, a fin de generar información con mayor grado de detalle, se busca conocer el comportamiento en resolución horaria del P y H. El cálculo de los índices a nivel mensual ( $\mathrm{P}, \mathrm{THI}$, H y TCl) se realizó con base en las estadísticas climatológicas de la estación Iguazú Aero para el período 1981-2010, proporcionadas por el Servicio Meteorológico Nacional. Los índices $\mathrm{P}$ y $\mathrm{H}$ en resolución horaria se calcularon a partir de datos meteorológicos de la misma estación para el período 2001-2010, obtenidos del sitio Meteomanz.com. Los resultados obtenidos muestran que la componente termo-higrométrica es la de mayor peso en el destino analizado, lo cual es coherente con sus características climáticas. La aplicación de los índices permitió determinar los momentos del año y horas del día más benignas para la realización de prácticas turístico-recreativas al aire libre.

PALABRAS CLAVE: Clima, Turismo; Índices de confort bioclimático, Índices de confort climático-turísticos; Faceta térmica del clima.

\section{CLIMATE COMFORT AND TOURISM. APPLIED STUDY AT DIFFENT TEMPORAL-RESOLUTION LEVES IN PUERTO IGUAZÚ, ARGENTINA}

ABSTRACT: The climate is a factor that conditions tourist-recreational practices in outdoor spaces. Thermal well-being and comfort are visitor demands that can be evaluated through the thermal facet of the climate. This paper is applied to Puerto Iguazú, a destination with a humidsubtropical climate. It is structured based on two objectives, on one hand, comparing three bioclimatic indices: Thermo-anemometric Complex (P), Thermo-hygrometric Complex (THI) and Humidex (H), versus a climatic-tourist index: Tourism Climate Index (TCI) to establish which one offers a better adjustment to the reality studied. On the other hand, in order to generate information with a greater degree of detail, we seek to know the behavior in hourly resolution of the $\mathrm{P}$ and $\mathrm{H}$. The calculation of the indices at a monthly level $(\mathrm{P}, \mathrm{THI}, \mathrm{H}$ and $\mathrm{TCl})$ was carried 
out based on the climate statistics of the Iguazú Aero station for the 1981-2010 period, provided by the National Meteorological Service. The $\mathrm{P}$ and $\mathrm{H}$ indices in hourly resolution were calculated from meteorological data from the same station for the 2001-2010 period, obtained from the website Meteomanz.com. The obtained results show that the thermo-hygrometric component is the one with the greatest weight in the analyzed destination, which is consistent with its climatic characteristics. The application of the indices made it possible to determine the most benign moments of the year and hours of the day for carrying out tourist-recreational activities in outdoor.

KEY WORDS: Climate; Tourism; Bioclimatic comfort indices; Climate-Tourism comfort indices; Thermal facet.

\section{INTRODUCCIÓN}

El interés por conocer las relaciones entre la actividad turística y los elementos climáticos es relativamente reciente. De acuerdo con de Freitas (2017), la Climatología del Turismo comenzó a desarrollarse entre las décadas de 1960 y 1970 durante el período denominado "revolución climática". A partir de los años 90 el abordaje del binomio clima-turismo empezó a profundizarse, de modo que las investigaciones se enfocaron entre otros temas en el tiempo y el clima como factores limitantes para la actividad (de Freitas, 2017 y Martínez Ibarra, 2006).

Uno de los acuerdos con respecto al binomio es la sensibilidad del turismo ante las condiciones climáticas. En este sentido, Smith (1993) realizó una diferenciación entre dependencia climática y sensibilidad meteorológica. El primer caso refiere a aquellas actividades cuya práctica depende, en gran medida, de las condiciones climáticas de sitio, las cuales a su vez marcan la estacionalidad. Por ejemplo, la presencia de determinada cantidad y calidad de la nieve para la realización de deportes de invierno; o de cierta temperatura del aire, velocidad del viento y cantidad de horas de sol para el turismo de sol y playa. Por su parte, el segundo caso, se manifiesta en aquellos destinos cuya oferta turística principal no depende de las condiciones climáticas, aunque el tiempo meteorológico puede incidir en el tipo de actividades a desarrollar en los espacios al aire libre. Por ejemplo, senderismo, trekking, escalada y ciclismo.

Las actividades realizadas en espacios al aire libre están más expuestas a la incidencia de los elementos climáticos puesto que no es posible controlar su comportamiento. Por ello, cobra relevancia lo que de Freitas $(1990,2001,2005)$ denomina facetas o atributos del clima, las cuales se clasifican en i) térmica, ii) física y iii) estética. La primera (i) se relaciona con los elementos físicos (tasa metabólica, nivel de arropamiento, nivel de actividad) y climáticos (temperatura del aire, humedad relativa, viento y radiación solar) que influyen en el estado térmico y de confort del organismo (Gómez Martín, 2017). La segunda (ii) se basa en los elementos que afectan directa o indirectamente a la práctica turística, en tanto suponen algún grado de peligro y/o molestia. Es el caso de las precipitaciones, el viento y la radiación ultravioleta. Por último, la tercera faceta (iii) se asocia con la calidad de la experiencia turística, por lo que los elementos más vinculados son la cobertura nubosa, la insolación, la visibilidad y la duración del día. Estas tres facetas se relacionan con las exigencias del visitante para la práctica del turismo, definidas por Besancenot (1991) y retomadas años más tarde por otros autores como Gómez Martín (1999, 2005, 2017); Lam González, León González y de León Ledesma (2018) y Martínez Ibarra y Pardo Martínez (2017). Estas exigencias son tres: la seguridad, el disfrute y el confort y la salud. Este trabajo se centra en la faceta térmica del clima, la cual se asocia con la exigencia del confort por parte del visitante.

El concepto de confort térmico refiere a aquellas condiciones del ambiente para las cuales el organismo mantiene al mínimo sus mecanismos de termorregulación, es decir que las ganancias y pérdidas de energía se mantienen en equilibrio y el cuerpo no se enfrenta a situaciones de estrés por frío o calor (Fernández García, 1996). A esta componente fisiológica se le agrega una psicológica, ya que la mayoría de las definiciones alude al estado de ánimo que expresa la sensación de bienestar con el ambiente al que están expuestas las personas (Ochoa de la Torre, Lovriha y Alpuche Cruz, 2009; Erazo, Garzón, Peña y Salazar, 2014). Las variables climáticas que inciden en la configuración del confort son la humedad del aire, la velocidad del viento, la radiación y la temperatura (Fernández García, 1996; Matzarakis, 2007; Toy, Yilmaz y Yilmaz, 2007; Scott, Gössling y de Freitas, 2008). En síntesis, el ambiente térmico tiene efectos sobre la salud, la seguridad y el bienestar.

Para la actividad turística, el confort es una variable que permite conocer e informar al visitante sobre el momento del año más benigno para viajar y planificar tentativamente las actividades en el destino. Nastos y Matzarakis (2019) afirman que la decisión de viaje está asociada, por un lado, al potencial físico y económico de los individuos y por otro, a sus preferencias moldeadas a partir de múltiples elementos, entre ellos el clima del lugar de residencia y las características del destino deseado (paisaje, clima y cultura). En relación con ello, cuanto más opuestas son las condiciones climáticas 
entre origen y destino, es probable que el cuerpo se exponga a situaciones de estrés por frío o calor; cuya incidencia en la salud depende de las características individuales. Por lo tanto, algunos grupos poblacionales pueden ser más sensibles al estrés térmico que otros (Katerusha y Matzarakis, 2015; Rutty y Scott, 2016; Salata, Golasi, Proietti y de Lieto Vollaro, 2017). Por otro lado, desde el punto de vista de la gestión de los destinos, la disponibilidad de información sobre el confort puede contribuir a diversificar la oferta turística y mitigar la estacionalidad cuando las condiciones climáticas lo permitan (Rutty y Andrey, 2014; Rutty y Scott, 2016; Wilkins, de Urioste-Stone, Weiskittel y Gabe, 2017). En suma, el estudio de las tres facetas del clima es importantes para la actividad turística, sin embargo, si se pretende explorar las implicancias del clima a nivel fisiológico, cobra especial interés la faceta térmica.

La faceta térmica se aborda generalmente desde un enfoque cuantitativo, basado en la aplicación de modelos de balance térmico e índices bioclimáticos y climático-turísticos para cuantificar las sensaciones térmicas y definir los umbrales de confort para un determinado espacio-tiempo. Los índices se clasifican en directos (basados en mediciones directas de los parámetros ambientales), empíricos (fundados en el estrés objetivo o subjetivo) y racionales (centrados en el equilibrio térmico del cuerpo) (Nastos y Matzarakis, 2019). No obstante, en las últimas dos décadas los estudios también se desarrollaron en torno a la componente psicológica. En efecto, se ha expandido el enfoque de investigación mixto al combinar la aplicación de índices con cuestionarios a la demanda turística potencial y efectiva para determinar sus preferencias con respecto al clima y confort térmico in situ. Este tipo de trabajos realiza aportes con respecto a los ideales climáticos de un determinado segmento de la demanda y con relación a una modalidad turística concreta (Nikolopoulou y Steemers, 2003; Scott, Gössling y de Freitas, 2008; Lin y Matzarakis, 2011; Stewart, Lazo, Morss y Demuth, 2012; Rutty y Scott, 2015; 2016; Martínez Ibarra y Pardo Martínez, 2017; Martínez-Ibarra, Gómez-Martín, Armesto-López y Pardo-Martínez, 2019; Shang, Huang, Zhang y Chen, 2019; Torrens Calleja, 2020).

Por otro lado, los estudios centrados en la componente fisiológica son variados. Es frecuente la aproximación por medio de índices bioclimáticos cuyo grado de complejidad varía en función de la disponibilidad de datos climáticos, así como también de los objetivos del estudio y criterios del investigador. Otros estudios se basan en los algoritmos de los modelos de balance de calor. Por ejemplo, Mateeva (2001) utilizó el modelo MENEX (Man-Environmental Heat Exchange) para determinar las condiciones bioclimáticas de Bulgaria e identificar los espacios más favorables para el turismo durante el año. Por su parte, Matzarakis (2001; 2012) desarrolló el RayMan que permite estimar los flujos de radiación y el efecto de las nubes. Con base en ese modelo se pueden calcular diferentes índices bioclimáticos complejos que requieren datos de radiación que habitualmente no están disponibles en las estadísticas climatológicas. Uno de ellos, es la Temperatura Fisiológica Equivalente (PET por sus siglas en inglés), basado en el Modelo de Equilibrio Energético de Múnich para Individuos (MEMI por sus siglas en inglés). El PET es un índice ampliamente utilizado para determinar los umbrales termo-fisiológicos de confort (Potchter, Cohen, Lin y Matzarakis, 2018). Se ha aplicado en diferentes territorios y escalas. Por ejemplo, en las ciudades de Bursa (Turquía) (Çalışkan, Çiçek y Matzarakis, 2012), Haikou (China) (Shang et al., 2019), Isfahan (Irán) (Nasrollahi, Hatami y Taleghani, 2017) y Odessa (Ucrania) (Katerusha y Matzarakis, 2015). A escala media, en las regiones del lago Balatón (Hungría) (Németh, Matzarakis, Schlanger y Katona, 2008) y en la meseta tibetana de China (Li y Chi, 2013); y a menor escala en Croacia (Zaninović y Matzarakis, 2009), Milos (Nastos y Matzarakis, 2019) y Polonia (Błażejczyk y Matzarakis, 2007).

Otros trabajos han aplicado índices bioclimáticos diferentes del PET que varían su nivel de complejidad, como el Complejo Termo-higrométrico (THI), el Complejo Termo-anemométrico, el Humidex, el Predicted Mean Vote (PMV), el Universal Thermal Climate Index (UTCI) y el Relative Strain Index (RSI), entre otros (Batista Tamayo y Matos Pupo, 2004; Bustos y Piccolo, 2011; Rutty y Scott, 2014; Ahmadi y Ahmadi, 2017; Bistricean, Mihăilă y Liliana, 2017; Salata et al., 2017; Zare et al., 2018). El Tourism Climate Index (TCI), un índice climático-turístico, también es frecuentemente utilizado para conocer la aptitud climática anual de los destinos para el turismo puesto que contempla las tres facetas del clima. El TCl se aplicó en diferentes ciudades de Australia (Amelung y Nicholls, 2014), Canadá y Estados Unidos (Scott y McBoyle, 2001), en Chipre (Olya y Alipour, 2015); en ciudades y regiones de Irán (Farajzadeh y Matzarakis, 2009; Ramezani Gourabi y Palic, 2012; Hassan, Varshosaz y Eisakhani, 2015) y en la región del lago Sun Moon en Taiwán (Lin y Matzarakis, 2007), entre otros sitios.

La revisión de la literatura también permitió identificar que los estudios que evalúan la faceta térmica del 
clima, si bien se aplican en destinos de diferentes latitudes (Potcher et al., 2018) la mayoría pertenece al hemisferio Norte y en particular, aquellos realizados para las latitudes bajas tienden a concentrarse en destinos de sol y playa (Rutty y Scott, 2014, 2015, 2016; Abed y Matzarakis, 2018; Shang et al., 2019; Rutty et al., 2020). Esto representa una oportunidad para extender el análisis del binomio a destinos de climas subtropicales que no dependen necesariamente del clima, pero para los cuales la disponibilidad de datos termo-fisiológicos contribuya a mejorar la planificación turística y ofrecer información de interés para el visitante. Pues, en este tipo de clima, si bien los datos anuales permiten tener una noción acerca del momento más benigno para viajar; se debe advertir la existencia de variaciones de los parámetros climáticos en el ciclo anual. Ello implica que la medición de los efectos combinados de los mismos puede arrojar luz sobre la extensión o contracción de la temporada turística.

En la Argentina el abordaje del binomio clima-turismo es incipiente. Al momento no se ha conformado una base sólida y continua de estudios aplicados en diferentes niveles de resolución espacio-temporal. Por ello, este trabajo es de carácter exploratorio y se desprende de una investigación mayor que aborda las relaciones entre clima y turismo a nivel nacional. Esta presentación se centra en Puerto Iguazú, un destino turístico internacional de la Argentina de clima subtropical húmedo, cuya oferta turístico-recreativa se basa fundamentalmente en el contacto con la naturaleza y las actividades al aire libre.

De acuerdo con el tipo de clima del área de estudio, se presume que la componente térmica tiene un peso relativamente mayor que las facetas estética y física. Por ello, el primer objetivo propuesto en esta investigación es comparar tres índices bioclimáticos (centrados en la faceta térmica) versus un índice climático turístico que contempla las tres facetas del clima; a fin de determinar cuál presenta un mejor ajuste con respecto a sus resultados. Por otro lado, ante el interés sobre el componente fisiológico del confort, el segundo objetivo del trabajo es conocer su comportamiento horario a partir de dos índices bioclimáticos que miden los efectos combinados de la temperatura y la humedad, por un lado, y la temperatura y el viento por otro. Este tipo de información puede resultar un insumo para la planificación del destino y el diseño de material orientado a informar a los turistas sobre los momentos del día más adecuados para realizar actividades al aire libre, principalmente para los grupos poblacionales de riesgo.

\section{ÁREA DE ESTUDIO}

La ciudad de Puerto Iguazú ( $25^{\circ} 35^{\prime} 56^{\prime \prime}$ S- $54^{\circ} 34^{\prime}$ $24^{\prime \prime}$ ) ) se ubica en el extremo NO de la provincia de Misiones (Argentina) (Fig.1) y pertenece a la región Litoral de acuerdo con la regionalización turística del país propuesta en el Plan Federal Estratégico de Turismo Sustentable (PFETS) (MinTur, 2014). En relación con el clima, presenta una temperatura media anual superior a $20^{\circ} \mathrm{C}$ y una amplitud térmica anual de $9,6^{\circ} \mathrm{C}$. Las precipitaciones son abundantes y alcanzan los 2.000 $\mathrm{mm}$ anuales, distribuyendose durante todo el año, aunque el mes de octubre es el más lluvioso de la región (Fig. 2). Según Iglesias de Cuello (1981:193) la ciudad pertenece al conjunto de los “(...) climas cálidos y húmedos del norte y este de la Argentina, acordes con su posición cercana al trópico de Capricornio y abierta a la influencia de los vientos húmedos orientales (...)" provenientes del Anticiclón Permanente del Atlántico Sur. Los inviernos son suaves y el alto porcentaje de humedad del aire (80\% aproximadamente) reduce la oscilación diaria de la temperatura.

Estas condiciones climáticas favorecen el crecimiento de abundante vegetación, conocida como selva Misionera o Paranaense, que se extiende en la Argentina, Brasil y Paraguay. La misma presenta suelos ricos en materia orgánica y varios pisos de vegetación que le otorgan al área una gran riqueza florística que no se repite en otros espacios del país. La "tierra roja" o suelo laterítico es uno de los rasgos más llamativos del paisaje misionero, resultado del proceso de oxidación del basalto bajo condiciones de clima cálido y húmedo. El atractivo turístico principal de la región, como así también de la provincia, es el sistema Cataratas del Iguazú, en plena selva Paranaense, es un conjunto de $\mathbf{2 7 5}$ saltos de agua formados como consecuencia de los sucesivos mantos de basalto de diferente edad geológica. El salto de mayor altura se conoce como Garganta del Diablo y alcanza los $82 \mathrm{~m}$ de alto y $150 \mathrm{~m}$ de ancho, dispuesto en forma de $U$, es el más imponente y caudaloso del sistema. Este atractivo natural se declaró en 1984 Patrimonio Mundial por la UNESCO y en el 2012 se integró como una de las siete maravillas naturales del mundo (Ministerio de Ambiente y Desarrollo Sustentable, 2021). Las cataratas del Iguazú se encuentran dentro del Parque Nacional Iguazú que comprende poco más de 67.000 ha.

Puerto Iguazú actúa como principal centro de estadía para los visitantes, ya que la ciudad se encuentra a 17 $\mathrm{km}$ del parque. En este sentido, el destino se asocia principalmente con la modalidad del turismo de naturaleza, puesto que entre las principales motivaciones emerge el interés por el contacto con entornos naturales vírgenes 
FIGURA 1

LOCALIZACIÓN DEL ÁREA DE ESTUDIO
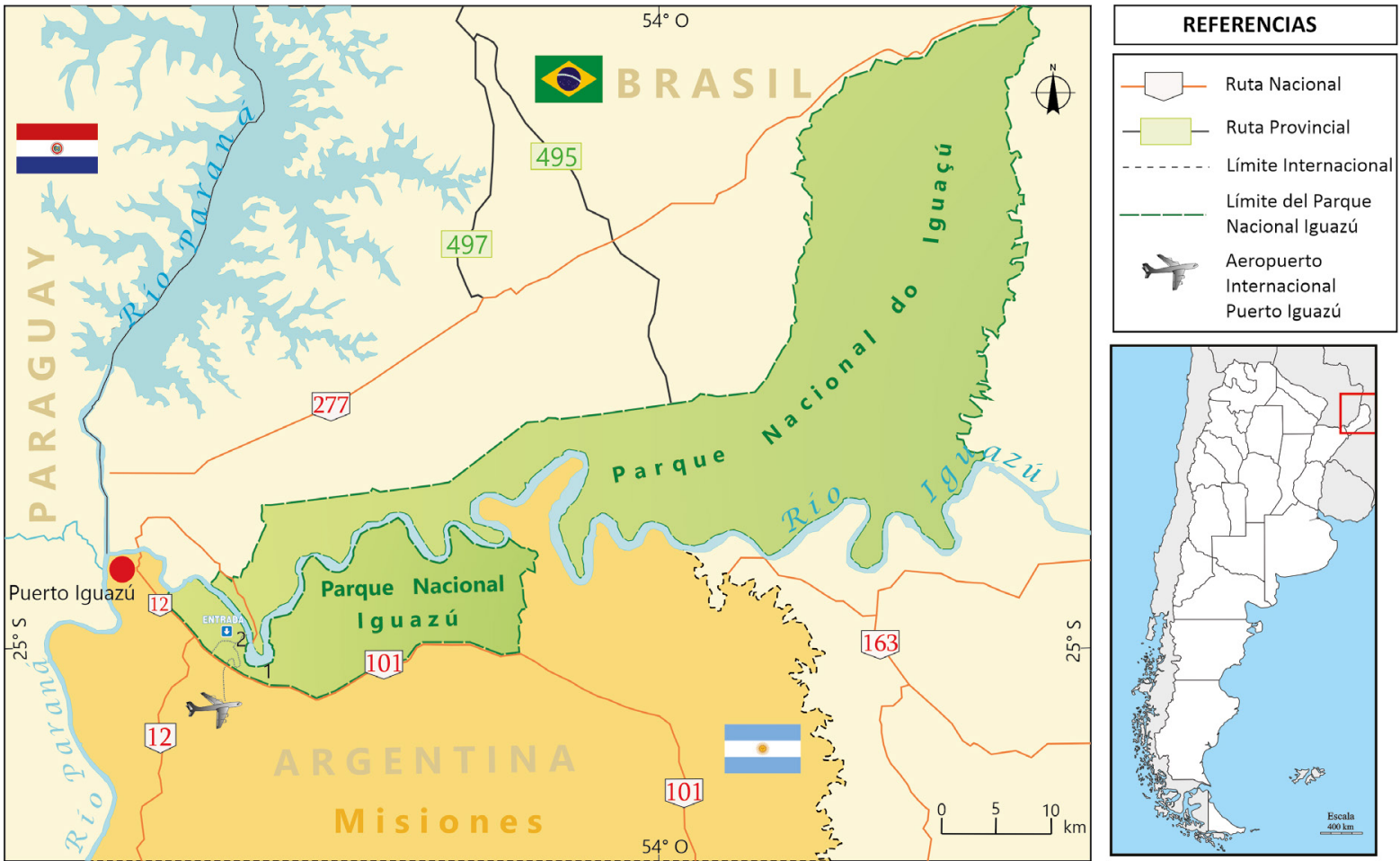

Fuente: Elaboración propia sobre la base de Google Earth (2016).

FIGURA 2

DISTRIBUCIÓN ANUAL DE LA TEMPERATURA Y LA PRECIPITACIÓN

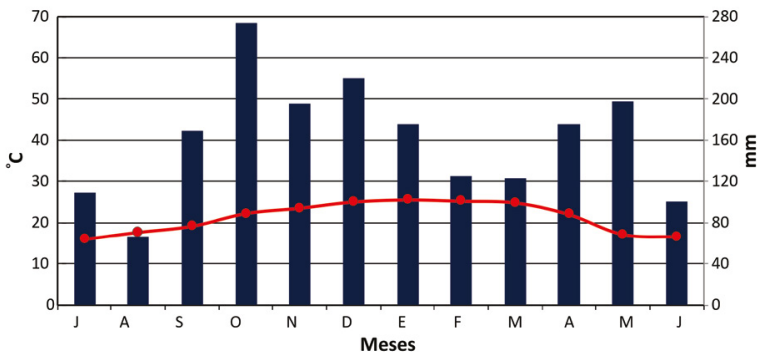

Fuente: Elaboración propia sobre la base de las estadísticas climatológicas del SMN (2001 - 2010).

o pocos modificados, la implicación del visitante con el espacio y el contacto con la comunidad local.

Para aproximar la distribución anual de la demanda turística del destino, se tomaron los datos del número de visitas al Parque Nacional Iguazú (Fig. 3). En términos medios, entre 2011 y 2019, se observa que los picos de demanda ocurren en enero y julio. En el primer semestre, febrero es el segundo mes de mayor afluencia, mientras que, en el segundo, son los meses de septiembre y octubre.
FIGURA 3

VISITAS Al PARQUe NaCIONAL IgUazú (2011-2019)

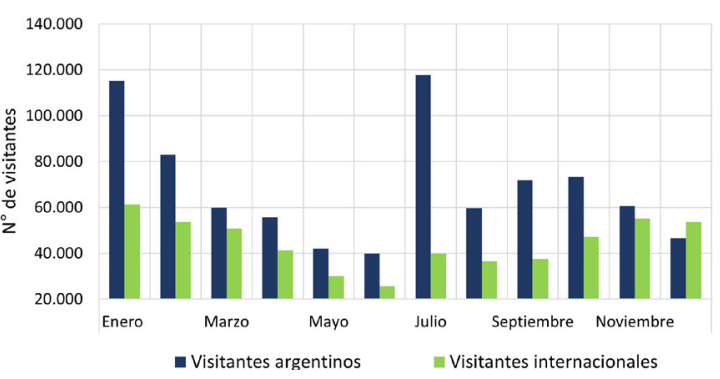

Fuente: Elaboración propia sobre la base de las estadísticas de la APN (2011-2019)

\section{METODOLOGÍA}

\section{Datos}

Este estudio se basa en las estadísticas climáticas mensuales del Servicio Meteorológico Nacional (SMN, Argentina) del período 1981-2010 y en los datos meteorológicos horarios obtenidos de la web Meteomanz.com para el período 2001-2010. En ambos casos los datos derivan de las mediciones realizadas por la estación meteorológica Iguazú Aero ( $25^{\circ} 44^{\prime} \mathrm{S}$; 
$\left.54^{\circ} 58^{\prime} 0 ; 270 \mathrm{~m}\right)$. La diferencia en el recorte temporal para la resolución mensual y horaria responde al acceso y disponibilidad sobre los datos. Por un lado, se utilizan promedios mensuales de temperatura media y máxima, humedad relativa media y mínima, precipitación, velocidad del viento y heliofanía y por otro, promedios horarios de temperatura media, humedad relativa y velocidad del viento.

\section{Instrumentos metodológicos: índices bioclimáticos y climático-turísticos}

En función de los objetivos y del alcance exploratorio del estudio se opta por la aplicación de cuatro índices directos debido a las siguientes ventajas: i) los datos de entrada están disponibles en una serie de tiempo relativamente larga para el área de estudio; ii) son índices aceptados y vigentes en la literatura y iii) el cálculo propiamente dicho es sencillo, al igual que iv) la interpretación de sus resultados. Tres son índices bioclimáticos centrados en la faceta térmica del clima: el Complejo Termo-higrométrico (THI), el Complejo Termo-anemométrico $(P)$ y el Humidex $(H)$; mientras que el cuarto es un índice climático-turístico que contempla las tres facetas del clima, el TCl. Todos ellos, se calculan a escala mensual para el período 1981-2010 y se comparan sus resultados conforme a una grilla de control para determinar la conveniencia de aplicación en destinos de características climáticas semejantes a Puerto Iguazú. Se opta comparar los índices bioclimáticos con uno climático-turístico para aproximar en el área de estudio si la incorporación de las tres facetas del clima versus la faceta térmica incide en el número de meses más benignos para la actividad turística.

Por otro lado, a fin de mejorar la comprensión en este trabajo sobre la componente fisiológica del confort en Puerto Iguazú, se amplía la resolución temporal para los índices $\mathrm{P}$ y H, calculándolos a nivel horario para el período 2001-2010. Se desestima el cálculo del $\mathrm{THI}$ dado que incorpora variables semejantes al H. A continuación, se presentan los índices y fórmulas utilizadas para su cálculo.

El THI combina la temperatura del aire y el porcentaje de humedad relativa alcanzada a la hora del máximo térmico. Este índice fue formulado por Thom en 1959 (Besancenot, 1991). La fórmula para su aplicación es la siguiente:

$$
\mathrm{THI}=\mathrm{T}-\left[0.55-\left(0.0055^{*} \mathrm{HR}\right) *(\mathrm{~T}-14.5)\right]
$$

Donde: T es la temperatura media y HR es la humedad relativa. El rango de confort está dado por las temperaturas comprendidas entre $15{ }^{\circ} \mathrm{C}$ y $20{ }^{\circ} \mathrm{C}$. Los rangos se definen en: Confortable cuando la puntuación del índice es menor a $20^{\circ} \mathrm{C}$; Ligeramente desconfortable cuando el puntaje oscila entre $20,1-25^{\circ} \mathrm{C}$ y Desconfortable ante una puntuación mayor a $25^{\circ} \mathrm{C}$.

El P evalúa la cantidad de calor perdida por el organismo en una unidad de tiempo a través de la combinación de la temperatura máxima y la velocidad del viento a la hora del máximo térmico. El P fue desarrollado por Siple y Passel en 1945 (Ramos, Gil, Campo y Tanana, 2018). La fórmula correspondiente se presenta a continuación:

$$
\mathrm{P}=\left(10^{*} \mathrm{~V}^{\wedge} 0,5+10,45-\mathrm{V}\right) *(33-\mathrm{Tmax})
$$

Donde $\mathrm{V}$ es la intensidad del viento en $\mathrm{m} / \mathrm{s}$ y Tmax es la temperatura máxima media en ${ }^{\circ} \mathrm{C}$. Este índice se expresa en $\mathrm{kcal} / \mathrm{m}^{2} / \mathrm{h}^{-1}$. Las condiciones óptimas se sitúan entre 301 y $603 \mathrm{kcal} / \mathrm{m}^{2} / \mathrm{h}^{-1}$; ante un valor de $\mathrm{P}$ $>603$ el sujeto debe comenzar a protegerse contra la pérdida de calor y el punto crítico se encuentra por debajo de las $1400 \mathrm{kcal} / \mathrm{m}^{2} / \mathrm{h}^{-1}$ ya que no puede evitarse el congelamiento de la piel desnuda. En sentido opuesto, cuando P es < 301 el cuerpo debe evitar el calentamiento (Besancenot, 1991).

El $\mathrm{H}$ indica la temperatura efectiva percibida por el sujeto ante determinadas condiciones de humedad y temperatura ambiente. Este índice fue desarrollado por el Weather Service of Environment Canada en 2001. La expresión matemática para su cálculo se presenta a continuación (Capelli de Steffens, Piccolo y Campo de Ferreras, 2005).

$$
\mathrm{H}=\mathrm{T}+5 / 9 *(\mathrm{e}-10) \quad[3]
$$

En este caso, $\mathrm{T}$ es la temperatura media y " $\mathrm{e}$ " es la presión de vapor. Los resultados se clasifican en función de los siguientes rangos: $20-29^{\circ} \mathrm{C}$ (Confortable); $30-39^{\circ} \mathrm{C}$ (Algún desconfort); $40-45^{\circ} \mathrm{C}$ (Gran desconfort), en este caso se sugiere evitar ejercicios físicos; $>45{ }^{\circ} \mathrm{C}$ (Peligro) y $>54^{\circ} \mathrm{C}$ (Peligro inminente de sufrir un infarto al corazón). Los tres índices bioclimáticos (THI, P, H) por definición son aplicables a la estación estival. No obstante, dado que Puerto Iguazú pertenece a la clasificación de clima subtropical se considera adecuado su cálculo para todos los meses del año.

$\mathrm{El} \mathrm{TCl}$ es un índice climático-turístico cuyo objetivo es determinar la aptitud climática mensual de un destino para el turismo (Mieczkowski, 1985). Se basa en cinco sub-índices que se calculan por separado a partir de una escala de valoración que varía entre 0 y 5 puntos, donde 5 es el valor óptimo. El TCl se calcula a partir de la siguiente suma ponderada:

$I C T=2 *((4 * C I D)+C I A+(2 * P P)+(2 * H E)+V)=100$ 
Dónde: CID es el Índice de Confort Diurno que evalúa, mediante las tablas de ASHRAE, el efecto combinado de la temperatura máxima media $\left({ }^{\circ} \mathrm{C}\right)$ y humedad relativa mínima media (\%). El CIA es el Índice de Confort Diario que combina la temperatura media del aire $\left({ }^{\circ} \mathrm{C}\right)$ y la humedad relativa media (\%). PP es el monto de precipitaciones $(\mathrm{mm}), \mathrm{HE}$ es el número efectivo de horas de brillo solar y $\mathrm{V}$ es la velocidad media del viento $(\mathrm{km} / \mathrm{h})$. Los resultados del TCl varían entre -20 y 100 puntos. Entre -20 y 39 las condiciones climáticas se definen "imposibles" para el turismo; entre 40 y 59 puntos las condiciones son "aceptables", entre 60 y 79 "buenas"; entre 80 y 89 son "excelentes" y los puntajes superiores a 90 son ideales (Mieczkowski, 1985).

\section{RESULTADOS}

\section{Análisis comparativo de índices bioclimáticos y cli- mático-turísticos de resolución mensual}

\section{Índices bioclimáticos: $P, H$ y THI}

De acuerdo con los resultados obtenidos de la aplicación de los índices $\mathrm{P}$, H y THI (Fig. 4a, 4b y 4c, respectivamente), los meses de confort térmico en Puerto Iguazú están comprendidos entre mayo y septiembre. Es decir que, en términos medios, el $40 \%$ del año presenta condiciones térmicas agradables, por lo cual se espera que el organismo no se enfrente a situaciones de estrés por calor debido al estado de neutralidad térmica. No obstante, como se mencionó en el apartado introductorio, esto queda sujeto en alguna medida a las condiciones fisiológicas de cada individuo, así como también al tipo de condiciones climáticas en las que se encuentra acostumbrado a desenvolverse. De acuerdo con Blasco Redondo (2012), el cuerpo tarda más en aclimatarse a realizar actividades deportivas o de exigencia física en ambientes cálidos que fríos, por lo cual es esperable que los turistas que proceden de climas fríos experimenten alguna sensación de disconfort durante los primeros días en el destino, con independencia del mes en que se realiza el viaje, tendiendo a agudizarse durante la época estival, momento en que se recrudecen las condiciones de desconfort y aumenta la afluencia turística.

Con respecto a los resultados obtenidos para cada índice, si bien son coherentes entre sí, se advierten diferencias. En el caso del P, durante la mayor parte del año se recomienda evitar el calentamiento, esto implica que el efecto del viento sobre el cuerpo no es refrigerante, puesto que cuando la temperatura del aire supera los $33^{\circ} \mathrm{C}$ (temperatura habitual de la piel desnuda), el viento genera sensación de sofoco ante la incapacidad del organismo de ceder el exceso de calor al ambiente por medio de la evaporación del sudor. En este sentido, los resultados obtenidos de la aplicación del $\mathrm{P}$ brindan un dato temo-fisiológico que no se deprende a priori de las características climáticas del área estudio. Es decir, que en este destino la componente termo-higrométrica no puede atenuarse por el efecto del viento. Además, en términos medios la velocidad del viento en $\mathrm{km} / \mathrm{h}$ no supera la categoría de brisa, o sea que el movimiento del aire se siente en la cara y se percibe el movimiento de las hojas de los árboles y las veletas comunes (SMN, 2021).

Por su parte, el $\mathrm{H}$ y el THI, pese a que se sustentan en parámetros climáticos análogos, presentan variaciones con respecto a los puntos críticos. Si se toman los resultados del $\mathrm{H}$, en ningún momento del año el organismo estaría expuesto a situaciones de gran desconfort, aunque en el período de octubre a agosto se experimenta algún desconfort. En oposición, el THI demuestra que el período entre noviembre y marzo es el más crítico, ya que se corresponde con el rango de temperaturas de desconfort.

Cuando las personas están expuestas a situaciones de estrés por calor en espacios al aire libre tienen menores posibilidades de atenuar esa condición que en las situaciones de estrés por frío. En el último caso el grado de arropamiento, la incorporación de calorías por medio de los alimentos y bebidas calientes y el aumento del nivel de actividad contribuyen a aumentar la producción de calor y en efecto, lidiar contra el frío. Sin embargo, en el primer caso se sugiere que el nivel de arropamiento sea bajo (0,5 clo: ropa interior corta, pantalones livianos de algodón, camisa o remera de manga corta de algodón y cuello abierto), priorizando la permeabilidad de los tejidos y el uso de prendas holgadas que favorezcan la circulación interior del aire (Tudela, 1982). Además, de acuerdo con la producción de calor metabólica según el nivel de actividad, en los ambientes cálidos y cargados de humedad se recomendaría que las personas se mantengan en estado de reposo o trabajo ligero (sentado con movimiento moderado de brazos y tronco o de pie solo con movimiento ligero de brazos). No obstante, las actividades turístico-recreativas relacionadas con el truismo de naturaleza demandan mayor esfuerzo físico (trabajo moderado a intenso) y, en consecuencia, el cuerpo tiene mayor exposición al estrés por calor. Estas son consideraciones a evaluar en los casos de los grupos poblacionales de riesgo, no solo desde el 
punto de vista etario (niños y adultos mayores), sino también por aquellos con patologías cardiovasculares y con sobrepeso.

FIGURA 4

ÍNDICES BIOCLIMÁTICOS APLICADOS A PUERTO IgUAZÚ (1981-2010)
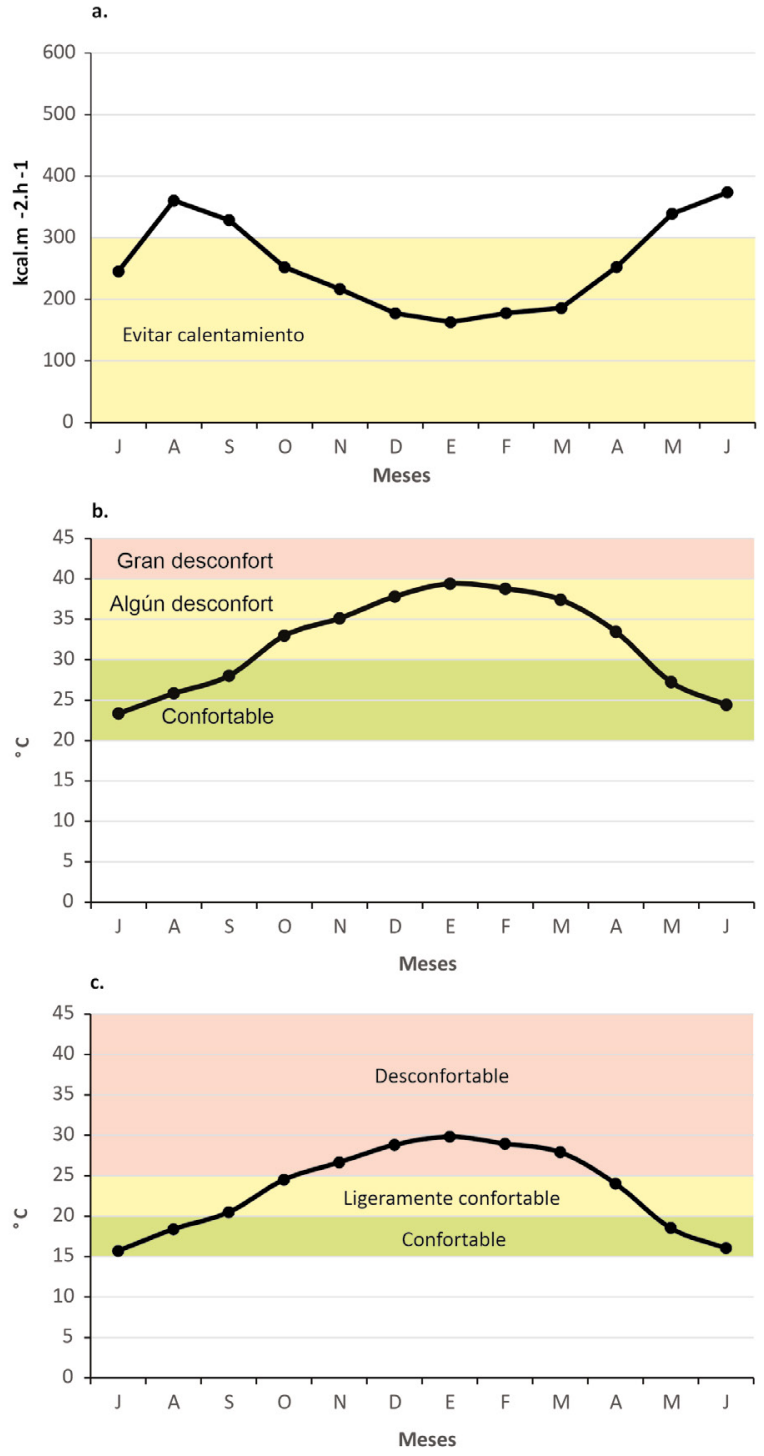

La figura presenta los tres índices bioclimáticos calculados en resolución mensual para Puerto Iguazú (período 1981-2010). La figura 4a presenta la curva del P, la $4 \mathrm{~b}$ el $\mathrm{H}$ y la 4c el THI. Elaboración propia sobre la base de datos del SMN (1981-2010).

\section{Índice climático-turístico: TCI}

En función de los puntajes obtenidos para el TCI (Fig. 5), el mes de enero es el único con características climáticas desfavorables para el turismo, aunque es uno de los dos momentos del año en el que se alcanza el pico en términos de afluencia de visitantes, como se mencionó en la caracterización del área de estudio. La categoría descriptiva correspondiente a enero se atribuye al efecto combinado de la temperatura y humedad y al puntaje relativamente bajo de los subíndices HE y PP. Conforme con el criterio de Mieczkowski (1985), para las prácticas turístico-recreativas se valora positivamente la cantidad de horas de brillo solar y la ausencia de precipitaciones. Sin embargo, en el caso de Puerto Iguazú se registran pocas horas en promedio de brillo solar debido al efecto de la nubosidad y un monto de precipitación superior a $150 \mathrm{~mm}$ medios mensuales. Solo tres meses del año evidencian condiciones buenas (mayo, julio y septiembre) para la actividad turísticas y solo uno se califica como muy bueno (agosto). Sin embargo, los meses restantes caen dentro de la zona de confort aceptable para el turismo.

FIGURA 5

TCI MEDIO MENSUAL APLICADO A PUERTO IgUAZÚ (1981-2010)

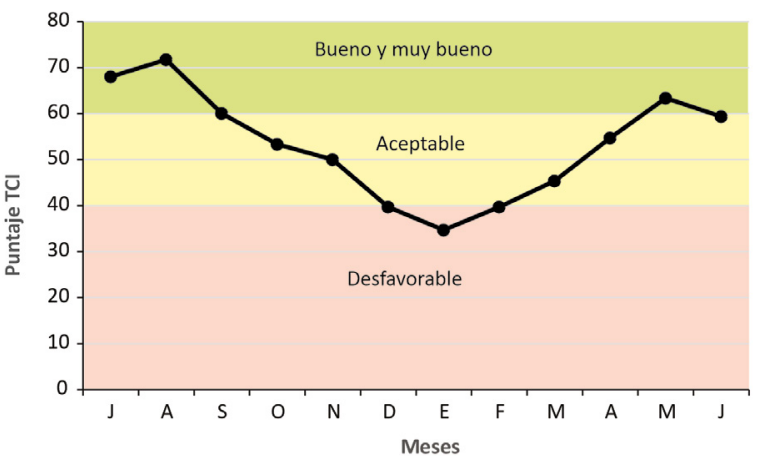

Fuente: Elaboración propia con base en las estadísticas climáticas del SMN (1981-2010)

Es interesante mencionar que el $\mathrm{TCl}$ es un índice discutido en la literatura especializada, aunque también resulta una de las aproximaciones más utilizadas para determinar la aptitud climática de un destino para el turismo. Entre las principales críticas, se establece que es un índice basado en la experticia del investigador ya que la valoración de cada subíndice se pondera de acuerdo con la revisión bibliográfica y opinión de Mieczkowski (1985) (Rutty et al., 2020). También se señala que es un índice general que no contempla los efectos de la radicación de onda corta y de onda larga (Millán López, 2017; Cheng y Zhong, 2019). No obstante, a los fines de este estudio, se consideró conveniente su utilización para contrastar los resultados con índices bioclimáticos centrados en la faceta térmica del clima, ya que el $\mathrm{TCl}$ i) incorpora las tres facetas del clima, de modo que considera la precipitación (faceta física), fenómeno muy relevante en el área de 
estudio; ii) emplea datos fácilmente asequibles en las estadísticas climatológicas del área de estudio y a su vez, iii) los resultados pueden interpretarse rápida y fácilmente por cualquier usuario no especializado (Perch-Nielsen, Amelung y Knutti, 2010; Scott, Rutty, Amelung y Tang, 2016).

\section{Comparación de índices bioclimáticos versus climá- tico-turísticos}

Los resultados obtenidos a partir de los cuatro índices a nivel mensual reflejaron consistencias en términos generales con la realidad esperada para un clima subtropical húmedo. Por ello, a continuación, se presenta una tabla comparativa a fin de establecer cuál es el más conveniente de aplicar en destinos de características semejantes a las de Puerto Iguazú, así como también, con realidades aproximadas en términos de la disposición de series temporales largas y completas de datos climatológicos.

La componente fisiológica del confort en Puerto Iguazú: aproximación a partir del $\mathbf{H}$ y el $\mathrm{P}$ en resolución horaria

La resolución mensual de los índices bioclimáticos como climático-turísticos brindan una aproximación sobre las temporadas más convenientes para realizar un viaje con fines turísticos y su variabilidad en términos del confort o aptitud climática a nivel intranual.
En efecto, su utilidad para el turista se remite principalmente a la fase de planificación y primeras decisiones relativas al viaje. En este sentido, cuando el visitante se encuentra efectivamente en el destino requiere información de confort climático en otra escala de resolución temporal (Gómez Martín, Armesto López y Martínez Ibarra, 2014; Millán López y Fernández García, 2018). En la actividad turística se valoran principalmente el nivel de resolución diario y horario, puesto que ofrecen mayor grado de detalle para planificar las actividades a realizar en entornos al aire. Por esto, con el objetivo de establecer una primera aproximación a la generación de este tipo de datos y focalizando en la componente fisiológica del confort, se presentan los índices $\mathrm{P}$ y $\mathrm{H}$ a nivel horario (Fig. 6). Determinar los rangos horarios más agradables, desde el punto de vista térmico, es relevante en función del tipo de actividades más frecuentes, entre las que se pueden mencionar: senderismo, trekking, ciclismo, navegación sobre el río Iguazú y caminatas por las pasarelas del Parque Nacional Iguazú.

En el primer trimestre del año (enero-marzo) se evidencia para el caso del $\mathrm{P}$ que, desde la primera hora de la mañana hasta las 21 horas, el efecto combinado de la temperatura del aire y la velocidad del viento genera alguna incomodidad para el organismo. Prácticamente durante todo el día las personas deberían evitar exponerse al calor o recurrir a alternativas para mitigar el estrés térmico (hidratarse constantemente,

TABLA 1

\begin{tabular}{|c|c|c|c|c|c|}
\hline & $\mathrm{P}$ & $\mathrm{H}$ & THI & $\mathrm{TCl}$ & Observaciones \\
\hline $\begin{array}{l}\text { Los resultados obtenidos son coherentes con } \\
\text { la realidad esperada. }\end{array}$ & $\checkmark$ & $\checkmark$ & & $\boldsymbol{v}$ & \\
\hline \multicolumn{6}{|l|}{ Período de confort (por trimestre) } \\
\hline \multicolumn{6}{|l|}{ Enero-marzo } \\
\hline Abril-junio & $\boldsymbol{v}$ & $\boldsymbol{\nu}$ & $\checkmark$ & $\checkmark$ & \\
\hline Julio-septiembre & $\checkmark$ & $\boldsymbol{V}$ & $\boldsymbol{v}$ & $\boldsymbol{v}$ & \\
\hline Octubre-diciembre & & & & $\boldsymbol{v}$ & \\
\hline \multicolumn{6}{|l|}{$\begin{array}{l}\text { Elementos climáticos considerados para su } \\
\text { cálculo }\end{array}$} \\
\hline Temperatura & $\checkmark$ & $\checkmark$ & $\boldsymbol{v}$ & $\boldsymbol{v}$ & \\
\hline Humedad relativa & & $\checkmark$ & $\checkmark$ & $\boldsymbol{v}$ & \\
\hline Velocidad del viento & $\boldsymbol{v}$ & & & $\boldsymbol{v}$ & \\
\hline Precipitación & & & & $\boldsymbol{v}$ & \\
\hline Heliofanía & & & & $\boldsymbol{v}$ & \\
\hline $\begin{array}{l}\text { Datos de entrada asequibles en estadísticas } \\
\text { climatológicas. }\end{array}$ & $\checkmark$ & & $\checkmark$ & $\checkmark$ & $\begin{array}{l}\text { En el caso del TCI la heliofanía puede resultar uno } \\
\text { de los elementos más complejos de obtener. }\end{array}$ \\
\hline Complejidad del cálculo & $\boldsymbol{v}$ & & $\boldsymbol{v}$ & $\boldsymbol{V}$ & $\begin{array}{l}\text { No se considera al H puesto que se debe calcular } \\
\text { la presión de vapor. }\end{array}$ \\
\hline \multicolumn{6}{|l|}{ Unidad de medida de los resultados } \\
\hline${ }^{\circ} \mathrm{C}$ & & $\checkmark$ & $\checkmark$ & & $\begin{array}{l}\text { Los resultados del TCI no tienen unidad de medida } \\
\text { (índice adimensional). }\end{array}$ \\
\hline $\mathrm{kcal} / \mathrm{m}^{2} / \mathrm{h}^{-1}$ & $\boldsymbol{v}$ & & & & \\
\hline
\end{tabular}


usar ropa liviana, permanecer a la sombra, intercalar actividades de trabajo moderado y ligero o en estado de reposo). Por su parte, la aplicación del H muestra que, si bien ninguna hora es térmicamente confortable, las condiciones de estrés por calor se recrudecen sensiblemente pasado el mediodía, debido a que es el momento de máxima insolación y, en efecto, del máximo térmico. Tanto los resultados del $\mathrm{P}$ como del $\mathrm{H}$ suponen que las condiciones del ambiente representan un peligro latente para los visitantes. Pues, de acuerdo con Hounam (1967), la sucesión de días cálidos se tolera con noches frescas que le permitan al organismo su recuperación, lo cual en este caso no ocurre.

En el segundo trimestre (abril-junio), el comportamiento de $\mathrm{P}$ y $\mathrm{H}$ mejora comparativamente con el primer período del año, debido fundamentalmente al descenso de la temperatura media. Por ejemplo, mientras que en abril se debe evitar el calentamiento entre las 12 y las 21 horas, en junio esta situación se acota a las 15 horas, momento en el que se alcanza el máximo térmico. En el caso de $\mathrm{H}$, se observa que ninguna hora del día es confortable por definición, pero tampoco en ningún momento se alcanzan condiciones de gran desconfort. En oposición, la marcha horaria del mes de junio solo presenta algún desconfort a las 15 horas. Esto alienta la programación de mayor número de actividades durante el transcurso de todo el día. El confort térmico horario del tercer trimestre (julio-septiembre), dado por $\mathrm{P}$ y $\mathrm{H}$, no presenta sustanciales diferencias con respecto al comportamiento del segundo trimestre. Aunque, entre las 15 y 18 horas, el cuerpo se puede ver expuesto a algún desconfort debido al progresivo incremento de la temperatura durante ese rango.

El último trimestre del año no es tan crítico como podría esperarse. Las horas en las que las personas deben evitar el calentamiento y experimentan algún desconfort se comprenden entre las 12 y 18 principalmente. Sin embargo, durante las horas de la noche mejoran las condiciones térmicas, por lo que se supone que el organismo tiene posibilidades de recuperarse para la siguiente jornada diurna de actividades.

\section{CONCLUSIONES}

Puerto Iguazú es un destino turístico internacional de la Argentina de clima subtropical húmedo. Es un centro de estadía a 17 km del Parque Nacional Iguazú, donde se encuentra el principal atractivo de la provincia y unos de los más importantes a nivel nacional: las Cataratas del Iguazú. La principal modalidad que se practica en el área de estudio es el turismo de natura- leza, por lo cual las actividades al aire libre tienen primordial relevancia. En este contexto y ante la exigencia de bienestar y confort por parte de los visitantes, se aplicaron tres índices bioclimáticos de distribución mensual centrados en la faceta térmica del clima $(\mathrm{P}, \mathrm{H}$ y $\mathrm{THI}$ ) y un índice climático-turístico (TCl) que incorpora las tres facetas (térmica, física y estética). De ello, se obtuvo que el período más benigno, desde el punto de vista fisiológico como climático-turístico, está comprendido entre mayo y septiembre. En términos medios mensuales, el $40 \%$ del año presenta condiciones climáticas agradables, de modo que los visitantes no se encuentran ante situaciones de estrés por calor ante su exposición en espacios al aire libre.

Los resultados obtenidos confirman el comportamiento esperado a priori para el confort en Puerto Iguazú. Sin embargo, se advierte, a partir del $\mathrm{P}$, que el viento no es una variable capaz de atenuar el efecto del componente termo-higrométrico, por el contrario, debido a las altas temperaturas, genera sofoco al impedir la liberación de calor del cuerpo al ambiente. Además, el H y el THI, pese a que se basan en parámetros climáticos análogos, evidencian variaciones con respecto a los meses calificados como críticos. Por último, de acuerdo con el $\mathrm{TCl}$, el único mes cuyas condiciones climáticas son desfavorables para el turismo es enero, mientras que mayo, julio, agosto y septiembre presentan condiciones buenas a muy buenas. Al contrastar la marcha anual de los índices con el comportamiento de las visitas al parque, se infiere que el clima no es una variable condicional para la elección del momento de viaje en este destino. Enero es uno de los dos picos de mayor número de visitas al parque y el mes más desfavorable desde el punto de vista térmico. Por esto, es importante que la oferta contemple estas condiciones a fin de mitigar en la medida posible las condiciones de desconfort.

Con respecto a la comparación entre los cuatro índices, se desprende que en el caso de Puerto Iguazú es conveniente aplicar índices como el $\mathrm{THI}$ o el $\mathrm{H}$, ya que consideran en su cálculo las variables de temperatura y humedad que definen principalmente su clima. En otras palabras, los resultados a nivel mensual evidencian la importancia del componente termo-higrométrico (faceta térmica) por sobre los demás componentes. Esto se fundamenta en la ausencia de variaciones significativas entre los resultados obtenidos del TCl con respecto al $\mathrm{P}, \mathrm{H}$ y THI. Pues, al incorporar las tres facetas del clima, se podría suponer que a partir de la aplicación del $\mathrm{TCl}$, el período más benigno podría extenderse o contraerse, situación que no se refleja 
FIGURA 6

ÍNDICES P Y H EN RESOLUCIÓN HORARIA (2001-2010)
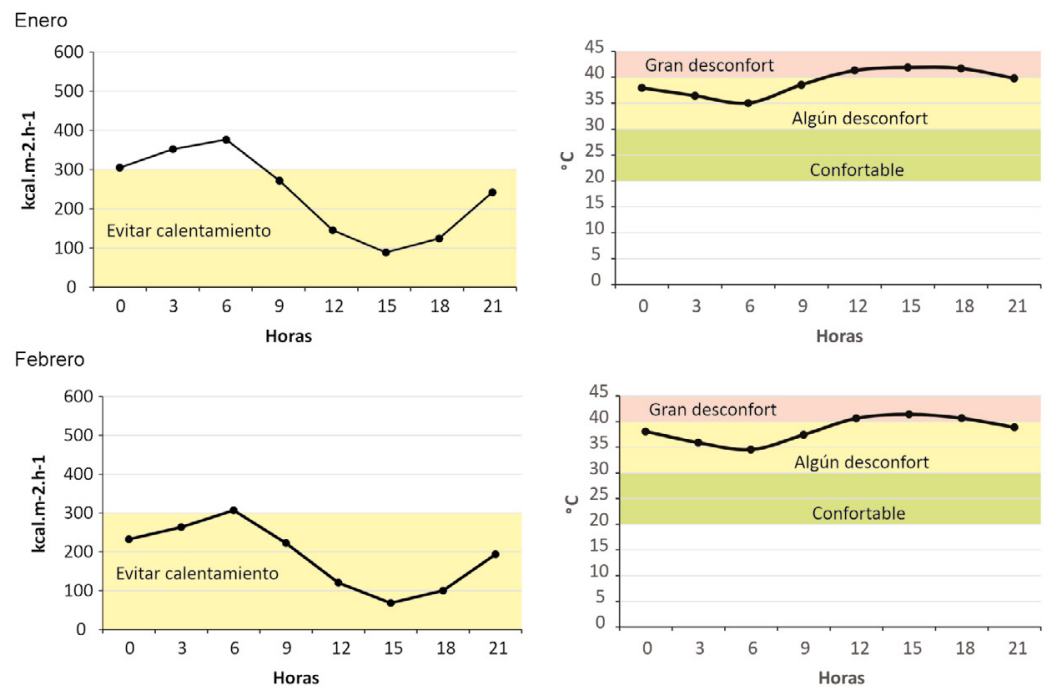

Marzo
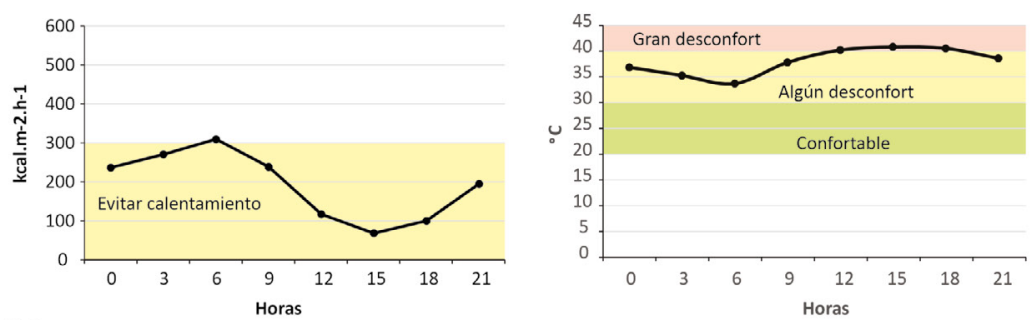

Abril
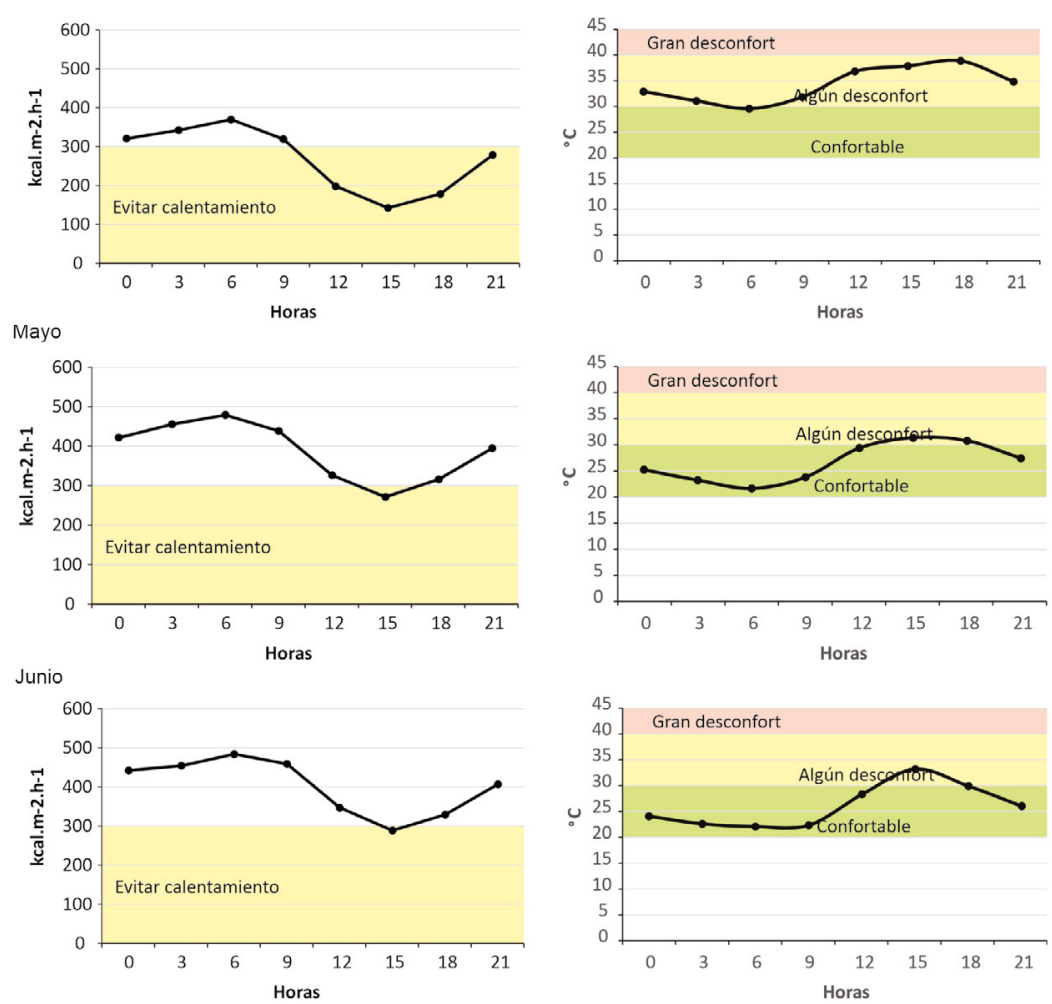
FIGURA 6

ÍNDICES P Y H EN RESOLUCIÓN HORARIA (2001-2010)

Julio
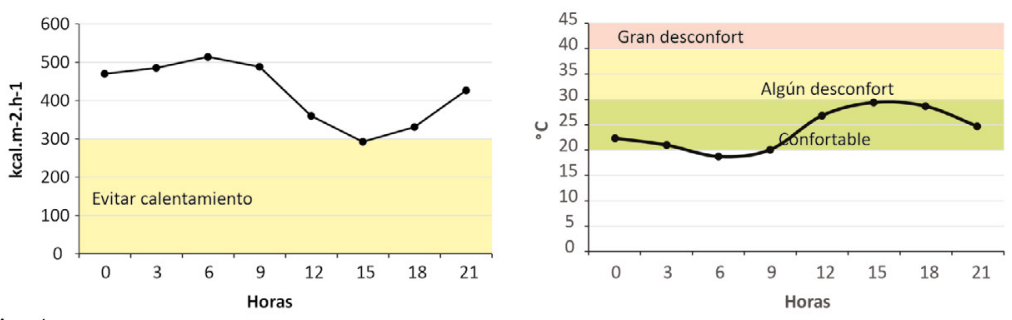

Agosto
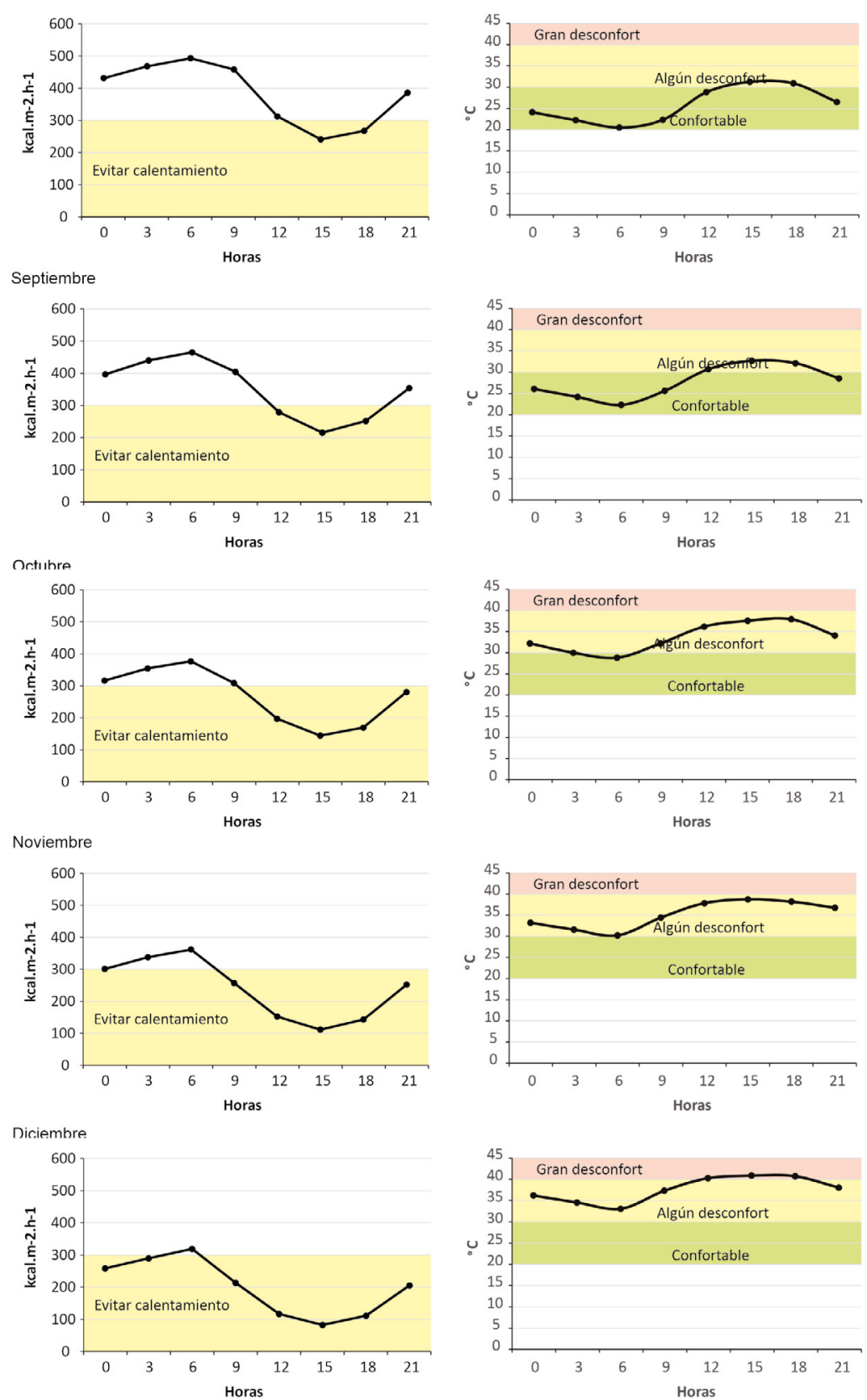

Fuente: Elaboración propia sobre la base de datos meteorológicos obtenidos de Meteomanz.com (2001-2010) 
en Puerto Iguazú. No obstante, también se reconoce que en la formulación del TCl el componente térmico es el que mayor peso relativo posee. En suma, los índices aplicados en resolución mensual son de sencilla aplicación y los datos de entrada para su cálculo están disponibles en las estadísticas climáticas. Esto puede promover el desarrollo de investigaciones exploratorias con respecto al binomio clima-turismo en destinos turísticos de características climáticas similares a las de Puerto Iguazú, donde la disponibilidad de datos climatológicos en diferentes niveles de resolución temporal sea asimétrica y discontinua.

Para la actividad turística es interesante generar datos de confort en resolución diaria y horaria ya que es un insumo concreto para planificar las actividades en el destino in situ. Por ello, se calcularon el $\mathrm{P}$ y $\mathrm{H}$ a nivel horario, de lo que se obtuvo que en los meses de desconfort (enero-febrero-marzo) durante todas las horas del día el cuerpo está expuesto a situaciones de estrés por calor, alcanzando el pico de mayor desconfort entre las 12 y 18 horas. Sin embargo, esto no se interpreta desde una concepción determinista, sino como datos objetivos que indican la necesidad de tomar recaudos por los visitantes con respecto a su exposición al aire libre durante las horas críticas. Enfatizando principalmente en el peligro que representan las condiciones térmicas para los grupos poblacionales de riesgo. En contraparte, el mes de julio no presenta ningún horario del día en el que el cuerpo se exponga a situaciones de estrés por calor debido al efecto combinado de temperatura y humedad $(\mathrm{H})$ o temperatura y viento $(P)$. Esto ofrece la posibilidad realizar mayor cantidad de actividades al aire libre, considerando que es el segundo momento de mayor afluencia de visitantes y el más óptimo desde el punto de vista del confort fisiológico.

Los resultados alcanzados en este trabajo sientan un antecedente sobre el estudio preliminar del binomio clima-turismo en uno de los destinos de mayor importancia del país y la región turística Litoral. En este sentido, a fin de alcanzar en el mediano plazo un análisis más profuso e integral, es importante orientar los esfuerzos, en primer lugar, a obtener datos climatológicos a nivel diario y horario de series históricas superiores a 30 años, no solo para el área de estudio sino también para la región y el país. Esto dará lugar a evaluar las facetas del clima a partir de índices más complejos que permitan construir una base de datos de confort para el área de estudio y otros destinos turísticos nacionales, con el fin último de determinar el escenario actual y sus potenciales cambios conforme a las proyecciones de cambio climático.

\section{AGRADECIMIENTOS}

Los autores agradecen al Servicio Meteorológico Nacional por proveer las estadísticas climáticas necesarias para la realización de este estudio. El presente trabajo se desarrolla en el marco del Proyecto Grupo de Investigación (PGI): "Clima y Turismo en la Argentina" subsidiado por la Universidad Nacional del Sur (código: 24/ZG20).

\section{REFERENCIAS}

Abed, S., \& Matzarakis, A. (2018). Quantification of the Tourism Climate of Algeria Based on the Climate-Tourism-Information-Scheme. Atmosphere,9(7), 250-269. https://www.mdpi.com/2073$4433 / 9 / 7 / 250$

Ahmadi, H., \& Ahmadi, F. (2017). Mapping thermal comfort in Iran based on geostatistical methods and bioclimatic indices. Arabian Journal of Geosciences, 10(15), 1-12. http://dx.doi.org/10.1007/ s12517-017-3129-3.

Amelung, B., \& Nicholls, S. (2014). Implications of climate change for tourism in Australia. Tourism Management, 41, 228-244. http://dx.doi. org/10.1016/j.tourman.2013.10.002.

Batista Tamayo, L.M., \& Matos Pupo, F. (2004). Aptitud climática del destino turístico Jardines del Rey (Cuba). Los tipos de tiempo [Actas de congreso]. El clima, entre el mar y la montaña, IV Congreso de la Asociación Española de Climatología, Santander, España. Recuperado de http://aeclim. org/wp-content/uploads/2016/02/0055_PU-SAIV-2004-LM_BATISTA.pdf.

Besancenot, J.P. (1991). Clima y turismo. Barcelona, España: Masson.

Bistricean, P. I., Mihăilă, D., \& Liliana, G. L. (2017). Bioclimatic regionalization of Moldova west of the Prut River. Present Environment and Sustainable Development, 11(1), 45-54. http://dx.doi. org/10.1515/pesd-2017-0004.

Blasco Redondo, R. (2012). Aclimatación al ejercicio físico en situaciones de estrés térmico. Revisión. Archivos de Medicina del Deporte, 29(148), 621631. Recuperado de http://femede.es/documentos/Revision\%20Aclimatacion_621_148.pdf.

Błażejczyk, K., \& Matzarakis, A. (2007). Assessment of bioclimatic differentiation of Poland based on the human heat balance. Geographia Polonica, 80(1), 63-82. 
Bustos, M.L., \& Piccolo, C. (2011). Desconfort térmico en verano e invierno para la localidad de Pehuen Co, Argentina. Nimbus, (27-28), 63-76. Recuperado de https://ri.conicet.gov.ar/bitstream/handle/11336/22294/CONICET_Digital_Nro.25559. pdf?sequence $=1 \&$ isAllowed $=y$.

Çalışkan, O., Çiçek, İ., \& Matzarakis, A. (2012). The climate and bioclimate of Bursa (Turkey) from the perspective of tourism. Theoretical and applied climatology, 107(3), 417-425. http://dx.doi. org/10.1007/s00704-011-0489-6.

Capelli de Steffens, A., Piccolo, M.C., \& Campo de Ferreras, A.M. (2005). Clima urbano de Bahía Blanca. Bahía Blanca, Argentina: Dunken.

Cheng, QP., \& Zhong, FL. (2019). Evaluation of tourism climate comfort in the Grand Shangri-La region. Journal of Mountain Science, 16(6). https://doi. org/10.1007/s11629-018-5081-4.

de Freitas, C. R. (2005). The climate-tourism relationship and its relevance to climate change impact assessment. En C. M. Hall \& J. Higham (Eds.), Tourism, Recreation and Climate Change: International Perspectives (pp. 29-43). United Kingdom: Channelview Press.

de Freitas, C.R. (1990). Recreation climate assessment. International Journal of Climatology, 10(1), 89103. http://dx.doi.org/10.1002/joc.3370100110.

de Freitas, C.R. (2001), Theory, concepts and methods in tourism climate research. En A. Matzarakis \& C.R. de Freitas. (Eds.), Proceedings of the First International Workshop on Climate, Tourism and Recreation, International Society of Biometeorology, Freiburg, Germany. (12) (PDF) Climate and tourism - an Australian perspective (researchgate.n https://www.researchgate. net/publication/242100272_Climate_and_tourism_-_an_Australian_perspectiveet).

de Freitas, C.R. (2017). Tourism climatology past and present: A review of the role of the ISB Commission on Climate, Tourism and Recreation. International Journal of Biometeorlogy, 61(1), 107-114. http://dx.doi.org/10.1007/s00484-017-1389-y.

Erazo, M. Garzón, F. Peña, M. \& Salazar, K. (2014). Meteorología y Climatología. Confort climático [Archivo PDF]. https://www.researchgate.net/publication/312938719_CONFORT_CLIMATICO.

Farajzadeh, H., \& Matzarakis, A. (2009). Quantification of climate for tourism in the northwest of
Iran. Meteorological Applications: A journal of forecasting, practical applications, training techniques and modelling, 16(4), 545-555. http:// dx.doi.org/10.1002/met.155.

Fernández García, F. (1996). Manual de climatología aplicada. Clima, medio ambiente y planificación. Madrid, España: Síntesis.

Gómez Martín, M.B. (1999). La relación clima-turismo: consideraciones básicas en los fundamentos teóricos y prácticos. Investigaciones Geográficas, (21), 21-34.

Gómez Martín, M.B. (2005). Reflexión geográfica en torno al binomio clima-turismo. Boletín de la Asociación de Geógrafos Españoles, (40), 111-134.

Gómez Martín, M.B. (2017). Retos del turismo español ante el cambio climático. Investigaciones Geográficas, (67), 31-47. http://dx.doi.org/10.14198/ INGEO2017.67.02.

Gómez Martín, M.B., Armesto López, X.A., \& Martínez Ibarra, E. (2014). La información climático-meteorológica proporcionada al turista. Explorando el caso español. Anales de Geografía, 34(2), 97-117. http://dx.doi.org/10.5209/rev_ AGUC.2014.v34.n2.47074.

Hassan, E. M., Varshosaz, K., \& Eisakhani, N. (2015). Analysis and Estimation of Tourism Climatic Index ( $\mathrm{TCl}$ ) and Temperature-Humidity Index (THI) in Dezfoul [Discurso principal]. 4th International Conference on Environmental, Energy and Biotechnology, Madrid, España. http://www.ipcbee. com/vol85/rp008_ICEEB2015-C0015.pdf.

Hounam, C. E. (1967). Meteorological factors affecting physical comfort (with special reference to Alice Springs, Australia). International Journal of Biometeorology, 11(2), 151-162. https://doi. org/10.1007/BF01426842.

Iglesias de Cuello, A. (1981). Tipos de clima. en E. Chiozza y Figueira. (Dir.). Atlas Total, (14). Buenos Aires, Argentina: Centro Editor de América Latina.

Katerusha, O., \& Matzarakis, A. (2015). Thermal bioclimate and climate tourism analysis for Odessa, Black Sea. Geografiska Annaler: Series A, Physical Geography, 97(4), 671-679. http://dx.doi. org/10.1111/geoa.12107.

Lam González, Y., León González, C.J., \& de León Ledesma, J. (2018). El clima y su relación con las actividades, la fidelidad y la satisfacción del turista náutico que visita la Ruta del Sol (Ecuador). In- 
novar, 28(67), 41-57. http://dx.doi.org/10.15446/ innovar.v28n67.68612.

Li, R., \& Chi, X. (2013). Thermal comfort and tourism climate changes in the Qinghai-Tibet Plateau in the last 50 years. Theoretical and applied climatology, 117(3), 613-624. http://dx.doi.org/10.1007/ s00704-013-1027-5.

Lin, T. P., \& Matzarakis, A. (2007). Tourism climate and thermal comfort in Sun Moon Lake, Taiwan. International journal of biometeorology, 52(4), 281-290. http://dx.doi.org/10.1007/s00484-007-0122-7.

Lin, T. P., \& Matzarakis, A. (2011). Tourism climate information based on human thermal perception in Taiwan and Eastern China. Tourism Management, 32(3), 492-500. http://dx.doi.org/10.1016/j.tourman.2010.03.017.

Martínez Ibarra, E. (2006). La climatología turística: ¿Una rama del conocimiento emergente? Trabajo presentado en V Congreso AEC. Clima, sociedad y medioambiente, Zaragoza. http://aeclim.org/ wp-content/uploads/2016/02/0072_PU-SA-V2006-E_MARTINEZIBARA.pdf.

Martínez Ibarra, E., \& Pardo González, R. (2017). Preferencias climáticas para la práctica del senderismo en España: resultados preliminares. Revista Investigaciones Turísticas, (13), 164-177. http:// dx.doi.org/10.14198/INTURI2017.13.08.

Martínez-Ibarra, E.; Gómez-Martín, M.B.; ArmestoLópez, X.A., \& Pardo-Martínez, R. (2019). Climate preferences for tourism: perceptions regarding ideal and unfavourable conditions for hiking in Spain. Atmosphere, 10(11), 646-659. http:// dx.doi.org/10.3390/atmos10110646.

Mateeva, Z. (2001). Bioclimatic diversity of Bulgaria: a resource or a limiting factor of the recreation \& tourism? En A. Matzarakis \& C.R. de Freitas. (Eds.), Proceedings of the First International Workshop on Climate, Tourism and Recreation, International Society of Biometeorology. Freiburg, Germany. https://www.researchgate.net/publication/242100272_Climate_and_tourism_-_an_ Australian_perspective.

Matzarakis, A. (2001). Assessing climate for tourism purposes: Existing methods and tools for the thermal complex. En A. Matzarakis \& C.R. de Freitas. (Eds.), Proceedings of the First International Workshop on Climate, Tourism and Recreation, International Society of Biometeorology. Freiburg, Germany.
Matzarakis, A. (2007). Climate, thermal comfort and tourism. En B. B. Amelung, K. Blazejczyk, y A. Matzarakis. (Eds.). Climate Change and Tourism - Assessment and Coping Strategies (pp. 140-154). Maastricht Warsaw - Freiburg: Institute of Geography and Spatial Organization Polish Academy of Sciences.

Matzarakis, A. (2012). RayMan and SkyHelios Model - Two Tools for Urban Climatology [Archivo PDF]. https://www.urbanclimate.net/matzarakis/ papers/5_V_5_Matzarakis_RayMan_SkyHelios.pdf.

Mieczkowski, Z. (1985). The tourism climatic index: a method of evaluating world climates for tourism. Canadian Geographer/Le Géographe Canadien, 29(3), 220-233.

Millán López, A. (2017). Climatología del Turismo de la Comunidad Autónoma de Madrid (Tesis de doctorado). Universidad Autónoma de Madrid, Madrid.

Millán López, A., \& Fernández García, F. (2018). Propuesta de un índice climático-turístico adaptado al turismo de interior en la Península Ibérica: aplicación a la ciudad de Madrid. Investigaciones Geográficas, 70, 31-46. http://dx.doi.org/10.14198/ INGEO2018.70.02.

Ministerio de Ambiente y Desarrollo Sustentable. (2021). Parque Nacional Iguazú. Recuperado de https://www.argentina.gob.ar/parquesnacionales/iguazu.

MinTur. (2014). Plan Federal Estratégico de Turismo Sustentable (PFETS). Actualización 2014. Ministerio de Turismo de la Nación. Recuperado de https://www.argentina.gob.ar/sites/default/ files/plan_federal_estrategico_de_turismo_sustentable_2025.pdf.

Nasrollahi, N., Hatami, Z., \& Taleghani, M. (2017). Development of outdoor thermal comfort model for tourists in urban historical areas. A case study in Isfahan. Building and Environment, 125, 356-372. http://dx.doi.org/10.1016/j.buildenv.2017.09.006.

Nastos, P. T., \& Matzarakis, A. (2019). Present and Future Climate-Tourism Conditions in Milos Island, Greece. Atmosphere, 10(3), 145. http://dx.doi. org/10.3390/atmos10030145.

Németh, Á., Matzarakis, A., Schlanger, V., \& Katona, Á. (2008). Variations of thermal bioclimate and its influence to the tourism in the Lake Balaton Tourism Region (Hungary) [Archivo PDF]. Recuperado de https://www.urbanclimate.net/matzarakis/papers/ICB18_Tour-O03.pdf. 
Nikolopoulou, M., \& Steemers, K. (2003). Thermal comfort and psychological adaptation as a guide for designing urban spaces. Energy and Buildings, 35(1), 95-101. http://dx.doi.org/10.1016/S03787788(02)00084-1.

Ochoa de la Torre, J.M., Lovriha, I., \& Alpuche Cruz, M.G. (2009). Análisis del confort climático para la planeación de sitios turísticos [Artículo en actas de congreso]. Estrategias de Transformación y Gestión de la Ciudad: Perspectivas y Nuevas Tecnologías, 50 Congreso Internacional Ciudad y Territorio Virtual, Barcelona, España. Recuperado de https://upcommons.upc.edu/handle/2099/11586.

Olya, H. G., \& Alipour, H. (2015). Risk assessment of precipitation and the tourism climate index. Tourism Management, 50, 73-80. http://dx.doi. org/10.1016/j.tourman.2015.01.010.

Perch-Nielsen, S.L., Amelung, B., \& Knutti, R. (2010). Future climate resources for tourism in Europe based on the daily Tourism Climatic Index. Climatic Change, 103, 363-381. https://doi.org/10.1007/ s10584-009-9772-2.

Potchter, O., Cohen, P., Lin, T. P., \& Matzarakis, A. (2018). Outdoor human thermal perception in various climates: A comprehensive review of approaches, methods and quantification. Science of the Total Environment, 631, 390-406. http:// dx.doi.org/10.1016/j.scitotenv.2018.02.276.

Ramezani Gourabi, B., \& Palic, M. (2012). Recognition of monthly bioclimatic comfort with Tourism Climatic Index in Ramsar, Southwest of Caspian Sea, Iran. Landscape \& Environment, 6(1), 1-14. Recuperado de https://ojs.lib.unideb.hu/landsenv/ article/view/2288.

Ramos, M.B., Gil, V., Campo, A.M., \& Tanana, A. (2018). Índices de confort climático aplicados a la región del noroeste argentino. En C. Mikkelsen \& N. Picone (Comps.), Geografías del presente para construir el futuro. Miradas geográficas que contribuyen a leer el presente (pp. 733-745). Tandil, Argentina: Universidad Nacional del Centro de la Provincia de Buenos Aires. https://b38991bf-e520-4f46-b58c1b739d147699.filesusr.com/ugd/bb5093_54617c a7f3e841a6902be52f941a164f.pdf.

Rutty, M., \& Andrey, J. (2014). Weather forecast use for winter recreation. Weather, Climate, and Society, 6(3), 293-306. http://dx.doi.org/10.1175/ WCAS-D-13-00052.1.
Rutty, M., \& Scott, D. (2014). Thermal range of coastal tourism resort microclimates. Tourism Geographies, 16(3), 346-363. http://dx.doi.org/10.1080 /14616688.2014.932833.

Rutty, M., \& Scott, D. (2015). Bioclimatic comfort and the thermal perceptions and preferences of beach tourists. International Journal of Biometeorology, 59(1), 37-45. http://dx.doi.org/10.1007/ s00484-014-0820-x.

Rutty, M., \& Scott, D. (2016). Comparison of climate preferences for domestic and international beach holidays: A case study of Canadian travelers. Atmosphere, 7(2), 30. http://dx.doi.org/10.3390/ atmos7060080.

Rutty, M., Scott, D., Matthews, L., Burrowes, R., Trotman, A., Mahon, R., \& Charles, A. (2020). An Inter-Comparison of the Holiday Climate Index $(\mathrm{HCl}$ : Beach) and the Tourism Climate Index (TCl) to Explain Canadian Tourism Arrivals to the Caribbean, Atmosphere, 11(412), http://dx.doi.org/10.3390/ atmos11040412.

Salata, F., Golasi, I., Proietti, R., \& de Lieto Vollaro, A. (2017). Implications of climate and outdoor thermal comfort on tourism: the case of Italy. International Journal of Biometeorology, 61(12), 2229-2244. http://dx.doi.org/10.1007/s00484017-1430-1.

Scott, D. \& McBoyle, G. (2001). Using a 'Tourism Climate Index' to examine the implications of climate change for climate as a tourism resource. En A. Matzarakis \& C.R. de Freitas. (Eds.), Proceedings of the First International Workshop on Climate, Tourism and Recreation, International Society of Biometeorology. Freiburg, Germany.

Scott, D., Gössling, S., \& de Freitas, C. R. (2008). Preferred climates for tourism: case studies from Canada, New Zealand and Sweden. Climate Research, 38(1), 61-73. https://www.int-res.com/abstracts/ $\mathrm{cr} / \mathrm{v} 38 / \mathrm{n} 1 / \mathrm{p} 61-73 /$.

Scott. D., Rutty, M., Amelung, B., \& Tang, M. (2016). An Inter-Comparison of the Holiday Climate Index $(\mathrm{HCl})$ and the Tourism Climate Index $(\mathrm{TCl})$ in Europe. Atmosphere, 7(6), 80-96. https://doi. org/10.3390/atmos7060080.

Shang, C., Huang, X., Zhang, Y., \& Chen, M. (2019). Outdoor thermal comfort in a tropical coastal tourist resort in Haikou, China. Indoor and Built Environment, 29(5), 730-745. http://dx.doi. org/10.1177/1420326X19862337. 
Smith, K. (1993). The influence of weather and climate on recreation and tourism. Weather, 48(12), 398-404. http://dx.doi. org/10.1002/j.1477-8696.1993.tb05828.x.

SMN. (2021). ¿Cómo clasificamos la intensidad del viento? Recuperado de https://www.smn.gob. ar/noticias/\%C2\%BFc\%C3\%B3mo-clasificamos-laintensidad-del-viento.

Stewart, A. E., Lazo, J. K., Morss, R. E., \& Demuth, J. L. (2012). The relationship of weather salience with the perceptions and uses of weather information in a nationwide sample of the United States. Weather, Climate, and Society, 4(3), 172-189. http://dx.doi.org/10.1175/WCAS-D-11-00033.1.

Torrens Calleja, J. M. (2020). Percepción de la Temperatura Climatológica en el Turismo y en Residentes de la Isla de Mallorca. Advances in Social Sciences Research Journal, 7(1), 236-246. http:// dx.doi.org/10.14738/assrj.71.7662.

Toy, S., Yilmaz, S., \& Yilmaz, H. (2007). Determination of bioclimatic comfort in three different land uses in the city of Erzurum, Turkey. Building and Environment, 42(3), 1315-1318. http://dx.doi. org/10.1016/j.buildenv.2005.10.031.
Tudela, F. (1982). Bioclima y confort térmico. Edición Consejo Económico y Social (CEPAL). Recuperado de https://repositorio.cepal.org/ bitstream/handle/11362/24070/S8100572_ es. pdf?sequence $=1 \&$ isAllowed $=y$.

Wilkins, E., de Urioste-Stone, S., Weiskittel, A., \& Gabe, T. (2017). Weather sensitivity and climate change perceptions of tourists: A segmentation analysis. Tourism Geographies, 20(2), 273-289. http://dx.doi.org/10.1080/14616688.2017.1399 437.

Zaninović, K., \& Matzarakis, A. (2009). The bioclimatological leaflet as a means conveying climatological information to tourists and the tourism industry. International Journal of Biometeorology, 53(4), 369-374. http://dx.doi.org/10.1007/s00484-0090219-2.

Zare, S., Hasheminejad, N., Shirvan, H. E., Hemmatjo, R., Sarebanzadeh, K., \& Ahmadi, S. (2018). Comparing Universal Thermal Climate Index (UTCI) with selected thermal indices/environmental parameters during 12 months of the year. Weather and climate extremes, 19, 49-57. http://dx.doi. org/10.1016/j.wace.2018.01.004. 